\title{
Ein Plädoyer für starke Bürgerbeauftragte. Thüringer Erfahrungen nutzen
}

\author{
Joachim Linck
}

In der Bundesrepublik Deutschland erhielt Rheinland-Pfalz 1974 als erstes Bundesland einen Bürgerbeauftragten. ${ }^{1}$ In Schleswig-Holstein wurde zwar 1992 auch ein Bürgerbeauftragter eingeführt, dessen Wirken ist aber begrenzt auf die Behandlung „sozialer Angelegenheiten “2 . Zuletzt etablierte Mecklenburg-Vorpommern 1995 ein solches Amt. ${ }^{3}$ Die konstituierenden gesetzlichen Rechtsgrundlagen ${ }^{4}$ werden ergänzt durch einschlägige Vorschriften in den Geschäftsordnungen der jeweiligen Landtage.

Auf Bundesebene gab es insbesondere aufgrund der Beratungen in der am 8. Oktober 1970 eingesetzten „Enquetekommission für Fragen der Verfassungsreform “5 Diskussionen um die Einführung eines Bürgerbeauftragten. Die Bundestagsfraktion Bündnis 90/Die Grünen stellte 1996 einen dahingehenden Antrag, der jedoch scheiterte. ${ }^{6}$

Ganz anders verlief die Entwicklung in den meisten EU-Staaten und auf EU-Ebene, wo es seit 1995 einen europäischen Bürgerbeauftragten gibt. ${ }^{7}$ Dieser europäische Siegeszug des Bürgerbeauftragten- beziehungsweise Ombudsmannsgedankens hatte mit der Einrichtung eines „riksens ständers justitieombudsman “ in Schweden begonnen. ${ }^{8}$

\section{Zur Entwicklung des Petitionswesens in Thüringen}

Für die Diskussion um die Einführung starker Bürgerbeauftragter auch in Deutschland, die hiermit erneut angestoßen werden soll, dürften die Erfahrungen mit Bürgerbeauftragten und Petitionsausschüssen in Thüringen hilfreiche Anregungen bieten.

1 Errichtet auf einfach-gesetzlicher Grundlage durch das „Gesetz über den Bürgerbeauftragten des Landes Rheinland-Pfalz" vom 3. Mai 1974 (GVBl. 1974, S. 187); Lars Brocker, in: Christoph Grimm / Peter Caesar (Hrsg.), Verfassung für Rheinland-Pfalz, Wiesbaden 2001, Art. 90a, Rn. 8 ff.

2 „Gesetz über die Bürgerbeauftragte oder den Bürgerbeauftragten für soziale Angelegenheiten des Landes Schleswig-Holstein“ vom 15. Januar 1992 (GVOBl. 1992, S. 42).

3 Art. 36 Verf.; ausgefüllt durch das Petitions- und Bürgerbeauftragtengesetz vom 5. April 1995 (GVOBl. 1995, S. 190); Michael Sauthoff, in: Rainer Litten / Maximilian Wallerath (Hrsg.), Verfassung des Landes Mecklenburg-Vorpommern, Baden-Baden 2007, Art. 36.

4 Vgl. zu dieser Rechtslage Näheres bei Anne Debus, ThürVBl 2009, S. 78 ff.; Udo Kempf / Marco Mille, Rolle und Funktion des Ombudsmannes. Zur personalisierten parlamentarischen Verwaltungskontrolle in 48 Staaten, in: ZParl, 23. Jg. (1992), H. 1, S. $29-47$.

5 Vgl. Zwischenbericht BT-Drs. 6/3829, S. 29, S. 31, S. 33 ff.; Schlussbericht BT-Drs. 7/5924, S. 63 f.; vgl. dazu Norbert Achterberg / Martin Schulte, in: Hermann von Mangoldt / Friedrich Klein / Christian Starck (Hrsg.), Das Bonner Grundgesetz. Kommentar, Art. 45c, Rn. 4 ff.

6 BT-Drs. 13/3578; zu dieser Entwicklung: Anne Debus, a.a.O. (Fn. 4), S. 78; Stefan Beck / Klaus A. Klang, Anspruch und Wirklichkeit des Petitionswesens - neue Überlegungen zur Organisationsform, in: ZParl, 17. Jg. (1986), H. 1, S. 49 - 64, S. 63 f.

7 Zur europäischen Rechtslage vgl. Thomas Gnatzy, in: Bruno Schmidt-Bleibtreu / Hans Hofmann / Axel Hopfauf, Kommentar zum Grundgesetz, Art. 17, Rn. 9, 12; Udo Kempf / Marco Mille (Fn. 4), S. $29 \mathrm{ff}$.

8 Dazu näher: Jürgen Hansen, Die Institution des Ombudsmanns, Frankfurt am Main 1972, S. 1; Markus Franke, Ein Ombudsmann für Deutschland?, Frankfurt am Main u.a. 1999, S. 27. 
In der Verfassung des Freistaats Thüringen (ThürLV) war von Beginn an mit Artikel 14 das Petitionsrecht festgeschrieben, das durch Artikel 65 ThürLV mit der Errichtung eines Petitionsausschusses ergänzt wurde. Diese verfassungsrechtlichen Regelungen wurden ursprünglich einfach-gesetzlich durch das Petitionsgesetz vom 28. Juni $1994^{9}$ sowie die $\$ \$ 94$ ff. der Geschäftsordnung des Thüringer Landtags konkretisiert und ausgefüllt. Danach war allein der Petitionsausschuss für die Bearbeitung der Petitionen zuständig.

Auf der Grundlage eines von der Landesregierung am 7. Dezember 1990 eingebrachten Entwurfs, der in den parlamentarischen Beratungen durch den federführenden Petitionsausschuss grundlegende Änderungen erfuhr ${ }^{10}$, wurde mit dem „Gesetz über den Bürgerbeauftragten“ (BüG 2000) vom 25. Mai $2000^{11}$ ein Bürgerbeauftragter installiert. Dessen Aufgaben beschrieb $₫ 1$ Abs. 1; 2 Nr. 1 BüG 2000 unter anderem so: „Der Bürgerbeauftragte befasst sich mit den von den Bürgern an ihn herangetragenen Wünschen, Anliegen und Vorschlägen (Bürgeranliegen)“, die keine Petitionen sind. Darüber hinaus war er aber auch für die Bearbeitung von an ihn gerichteten Petitionen im Sinne von Artikel 14 ThürLV zuständig ( $\$ 1$ Abs. 2 Nr. 3; $\$ 5$ Abs. 1 Satz 1 BüG 2000). Er hatte sich um deren „einvernehmliche Erledigung zu bemühen“ (\$ 5 Abs. 1, Satz 2 BüG 2000). Sollte ihm das nicht gelingen, hatte er „den Petitionsvorgang mit einer Stellungnahme dem Petitionsausschuss zuzuleiten“ (\$ 5 Abs. 1 Satz 3 BüG 2000); der Petitionsausschuss entschied dann endgültig.

Über die einvernehmlich vom Bürgerbeauftragten erledigten Petitionen musste der Petitionsausschuss lediglich unterrichtet werden ( $\$ 6$ Abs. 1 Nr. 3 BüG 2000). Mit dieser so genannten Mainzer Lösung ${ }^{12}$ versuchte man dem in Rheinland-Pfalz und Thüringen gleichlautenden verfassungsrechtlichen Gebot zu entsprechen, dass der Petitionsausschuss Adressat der Petition bleiben muss, über die Petition zu entscheiden hat und nicht etwa der Bürgerbeauftragte Herr des Verfahrens ist. Demzufolge kann der Bürgerbeauftragte bei keiner Art der Erledigung von Petitionen, auch nicht bei den einvernehmlich erledigten, an die Stelle des Petitionsausschusses treten. ${ }^{13}$

Ein Blick in die Praxis der Landtage Rheinland-Pfalz und Thüringen, die der Autor aus eigener Erfahrung recht gut kennt, lehrt jedoch, dass es sich bei dieser Konstruktion, mit der man versuchte, das Verfassungsrecht zu wahren, um eine reine Fiktion handelt. Tatsächlich wurden und werden die von den Bürgerbeauftragten vorgelegten Listen über die von ihnen einvernehmlich erledigten Petitionen von den Petitionsausschüssen lediglich kommentarlos zur Kenntnis genommen („abgenickt“). Von einer „Entscheidung“ der Ausschüsse über diese Petitionen kann schon gar keine Rede sein. Aber selbst die Hilfskonstruktion von Anne Debus, die sich der Problematik durchaus bewusst ist, wonach „nur auf diese Weise - gegebenenfalls konkludent - die rechtlich letztlich nötige Sanktionierung der Erledigung der Petition durch das parlamentarische Gremium von statten gehen kann"14, vermag der Verfassung nicht ausreichend zu genügen. Die langen Listen der vom Bürgerbeauftragten einvernehmlich erledigten Petitionen sind in den Petitionsausschüssen ein reiner Durchlauf-

9 Vgl. GVBl. 1994, S. 797.

10 Vgl. Drs. 3/140; Beschlussempfehlung Drs. 3/660.

11 GVBl. 2000, S. 98.

12 Vgl. dazu $\$ 5$ Abs. 1, Satz 4 des rheinland-pfälzischen Bürgerbeauftragtengesetzes.

13 Vgl. zu dieser Problematik: Anne Debus, Kommentar zum Thüringer Bürgerbeauftragtengesetz, Wiesbaden 2006, $\$ 6$ Anm. 1.1; Paul J. Glauben, DRiZ 1991, S. 230; Lars Brocker, a.a.O. (Fn. 1), Art. 90a, Rn. 13; Hagen Matthes, Der Bürgerbeauftragte, Berlin 1981, S. 143 f.

14 Anne Debus, a.a.O. (Fn. 13), \$ 6 Anm. 1.1. 
posten. Die näheren Umstände der einvernehmlichen Erledigung werden nicht erfragt und schon gar nicht erörtert und somit auch nicht konkludent entschieden. Man ist einfach nur froh, dass der Bürgerbeauftragte dem Ausschuss die Petitionsbearbeitung abgenommen hat.

$\mathrm{Zu}$ der beschriebenen verfassungsrechtlichen Problematik trat eine weitere Schwierigkeit bei der praktischen Durchführung des Gesetzes hinzu: Der Bürgerbeauftragte durfte „Bürgeranliegen“, die keine Petitionen sind ( $\$ 1$ Abs. 1 Satz 1 und Abs. 2 Nr. 1 BüG 2000) eigenverantwortlich erledigen. Wie aber „Bürgeranliegen“ von Petitionen sauber abzugrenzen sind, blieb offen - trotz aller anerkennenswerten interpretatorischen Bemühungen und Kunstgriffe von Debus in ihrem Kommentar zu dem Bürgerbeauftragtengesetz. ${ }^{15}$

Von Seiten des Petitionsausschusses fand in der Praxis auch keinerlei Kontrolle gegenüber dem Bürgerbeauftragten zu dieser Kompetenzproblematik statt, so dass ein expansiv agierender Bürgerbeauftragter Petitionen durchaus weitgehend unbehelligt als „Bürgeranliegen" bearbeiten konnte.

Die dargestellten verfassungsrechtlichen Kompetenzprobleme sowie die Schwierigkeiten, Petitionen von Bürgeranliegen sauber abgrenzen zu können, mögen auch dazu beigetragen haben, das Bürgerbeauftragtengesetz im Jahr 2007 auf den parlamentarischen Prüfstand zu stellen. Der entscheidende Anstoß dazu ging jedoch von ganz anderen Motiven und Überlegungen auf Seiten der Mitglieder des Petitionsausschusses aus.

Je länger der Bürgerbeauftragte amtierte und seinen gesetzlichen Aufgaben gewissenhaft nachkam, umso stärker wuchs die Kritik an seiner Arbeit sowie an der Institution als solcher. Sie wurde insbesondere von Mitgliedern des Petitionsausschusses geübt. ${ }^{16}$ Diese weitgehend hinter vorgehaltener Hand geäußerte - Kritik entzündete sich nicht etwa daran, dass der Bürgerbeauftragte bei der Erfüllung seiner Aufgaben versagt oder nicht effektiv genug gearbeitet hätte. Ganz im Gegenteil: der Bürgerbeauftragte war bei der Erledigung von Petitionen im Vergleich zum Petitionsausschuss nicht nur erheblich schneller, sondern auch erfolgreicher, vielleicht aus Sicht seiner Kritiker eben zu erfolgreich. Diese Bilanz des Bürgerbeauftragten, die sich in der Öffentlichkeit zunehmend herumsprach, führte folgerichtig dazu, dass sich immer mehr Bürger mit ihren Petitionen an den Bürgerbeauftragten und nicht mehr an den Petitionsausschuss wandten. So stieg die Anzahl der Petitionen beim Bürgerbeauftragten vom Jahr 2001, dem Beginn seiner Tätigkeit, von 514 bis zum Jahr 2006 auf 921, während sie beim Petitionsausschuss in diesem Zeitraum bei gut 900 Eingängen stagnierte.

\section{Effizienzvergleich zwischen Bürgerbeauftragtem und Petitionsausschuss in Thüringen}

Ein Vergleich der Arbeit des Bürgerbeauftragten und des Petitionsausschusses in Thüringen im Hinblick auf die Erledigungsdauer und den Bearbeitungserfolg der an sie gerichteten Eingaben kann sich sinnvollerweise nur primär auf den Zeitraum zwischen 2001 und 2006

15 Vgl. ebenda, $\$ 1$ Anm. 1.3. und 1.4.

16 Hinweise darauf, dass Abgeordnete Ombudsmänner skeptisch als Konkurrenten empfinden, auch bei Ulrich Riehm / Christopher Coenen / RalfLindner / Clemens Blümel, Bürgerbeteiligung durch E-Petitionen: Analysen von Kontinuität und Wandel im Petitionswesen, Berlin 2009, S. 61; Annette Guckelberger, Aktuelle Entwicklungen im parlamentarischen Petitionswesen. Online-Petitionen, Öffentliche Petitionen, Landesrecht, Baden-Baden 2011, S. 20. 
beziehen, denn nur in dieser Zeit haben beide Institutionen parallel Petitionen bearbeitet. Bei den nachstehend aufgeführten Zahlen und Statistiken wird zwar nicht im Detail zwischen den Aufgaben des Bürgerbeauftragten („Bürgeranliegen“ mit und ohne Petitionsqualität $\$ 1$ Abs. 2 BüG2000) und denen des Petitionsausschusses („Bitten oder Beschwerden“ gemäß Art. 14 ThürLV - ebenso auch der bis 2007 geltende $\$ 1$ Abs. 1 PetG 1994; „Eingaben“ gemäß Art. 65 ThürLV) differenziert, aber trotz der unterschiedlichen, unpräzisen Terminologie lassen sich in der Sache - wie bereits dargestellt - keine signifikanten Unterschiede zwischen beiden Arten von Eingaben feststellen. Diese Feststellung trifft auch auf die sachlichen Gegenstände dieser Eingaben und deren Schwierigkeitsgrad zu. Alle öffentlich zugänglichen Jahresberichte des Bürgerbeauftragten und des Petitionsausschusses weisen aus, dass die Anliegen der Bürger im Wesentlichen dieselben Sach- und Problembereiche betrafen, also unabhängig davon ob sie an den Petitionsausschuss oder den Bürgerbeauftragten gerichtet wurden; insoweit lassen sich keine Präferenzen der Bürger zu Gunsten eines Petitionsadressaten feststellen.

Wenn im Folgenden ein Effizienzvergleich zwischen dem Petitionsausschuss und dem Bürgerbeauftragten hinsichtlich der Bearbeitungsdauer und der Erfolgsquoten vorgenommen wird, so lassen sich diese Bilanzen nicht mit letzter Genauigkeit durch Statistiken belegen. So fehlen in den Jahresberichten des Petitionsausschusses und des Bürgerbeauftragten einschlägige Aussagen zum Beispiel zur jeweiligen Erledigungsdauer vollständig, oder es mangelt bei den Erfolgsbilanzen an objektiven Vergleichsmaßstäben (Wann war eine Petition erfolgreich?).

Diese mangelhafte Transparenz liegt in der Konkurrenzsituation zwischen Petitionsausschuss und Bürgerbeauftragtem begründet, die in Thüringen, aber auch in Rheinland-Pfalz zu den auch sonst üblichen Spannungen zwischen konkurrierenden Institutionen führte. Beide kämpften um ihre öffentliche Reputation, um die Sicherung ihrer Kompetenzbereiche bis hin zu ihrer Daseinsberechtigung, was auch ihre jeweiligen Apparate mit einschloss. Keiner wollte Daten vorlegen, mit denen die eigene Stellung beschädigt werden könnte, und es wurden Daten herausgehoben, die die eigene Bedeutung in ein besonders gutes Licht setzen sollten.

Dennoch lassen die nachfolgenden Belege eine eindeutige Tendenz zu Gunsten des Bürgerbeauftragten erkennen, was im Hinblick auf dessen Arbeitsweise und die Akteure beider Institutionen keine große Überraschung sein dürfte. ${ }^{17}$ Aber dennoch wird dieses Bild von den Parlamenten entweder nicht wahrgenommen oder verdrängt, so dass daraus nicht die erforderlichen rechtspolitischen Konsequenzen gezogen werden. Dieses Politikum ist das zentrale Anliegen dieses Beitrags.

\subsection{Erledigungsdauer}

Die Erledigungsdauer betrug beim Bürgerbeauftragten in Thüringen bei über 80 Prozent der Bürgeranliegen, bei denen es sich ganz überwiegend um Petitionen handelte, im

$17 \mathrm{Zu}$ diesem Ergebnis kam im Übrigen auch ein Seminar an der Friedrich-Schiller-Universität Jena, in dem unter Leitung des Autors die Tätigkeitsberichte des Thüringer Petitionsausschusses und des Bürgerbeauftragten von 2001 bis 2006 ausgewertet und zudem die Fraktionssprecher in Petitionsfragen des Thüringer Landtages angehört wurden. 
Durchschnitt weniger als drei Monate. ${ }^{18}$ Die Erledigungsdauer von Petitionen in Petitionsausschüssen fällt erheblich ungünstiger aus. Die im Übrigen mit umfangreichen Statistiken versehenen Arbeitsberichte der Petitionsausschüsse erhalten allerdings bezeichnenderweise - im Gegensatz zu den Jahresberichten des Bürgerbeauftragten - gerade keine Angaben dazu, wie lange es vom Eingang einer Petition bis zu deren abschließender Bescheidung im Durchschnitt dauert. Alle Erfahrungen sprechen dafür, dass die durchschnittliche Bearbeitungsdauer von normalen, also nicht besonders komplizierten Petitionen im Petitionsausschuss bei circa neun Monaten liegt. Wie aus den verschiedenen Jahresberichten des Petitionsausschusses über die nicht erledigten Petitionen der jeweiligen Vorjahre ersichtlich, gab es sogar zahlreiche Petitionen, deren Bearbeitung länger als ein Jahr gedauert haben muss.

Petitionen können vom Bürgerbeauftragten erheblich schneller bearbeitet und entschieden werden, weil er flexibler und unbürokratischer handeln kann. Der Verfahrensgang zur Vorbereitung der Entscheidungen über Petitionen im Ausschuss ist in aller Regel ziemlich langwierig; das gilt insbesondere für Petitionen aus dem Kommunalbereich. Als erstes werden zu den Petitionen über die Staatskanzlei von den jeweils zuständigen Ministerien Stellungnahmen eingeholt, die von der Landesregierung dem Petitionsausschuss zugeleitet werden. Bis dahin kann es viele Monate dauern, insbesondere, wenn das zuständige Ministerium seinerseits Stellungnahmen von den ihm untergeordneten Behörden einholt, deren Kooperationsbereitschaft im Übrigen eher verhalten bis zögerlich als schnell und konstruktiv ist. Dieser Mangel wird in den Tätigkeitsberichten von allen Petitionsausschüssen in der Bundesrepublik Deutschland immer wieder moniert.

Dieser schleppende Verfahrensgang soll an folgendem Beispiel illustriert werden: Wenn in einer Petition über die mangelhafte Schneeräumung in einer Gemeinde Beschwerde geführt wird, dann läuft die von der Exekutive für den Petitionsausschuss eingeholte Stellungnahme über folgende Stationen: Staatskanzlei - Innenministerium - Landesverwaltungsamt - Landratsamt - Gemeinde sowohl hin als auch wieder zurück. Rückfragen und interne Abstimmungen können das Verfahren noch weiter verzögern. Nach dem Eingang der Stellungnahme beim Landtag wird von der Landtagsverwaltung dazu - gegebenenfalls nach weiteren Rückfragen und Recherchen -, eine Stellungnahme mit einer Beschlussempfehlung für die Abgeordneten angefertigt. Dann wird entschieden, auf welche Tagesordnung des Petitionsausschusses die Angelegenheit gesetzt wird, der in der Regel nur einmal im Monat, also circa elfmal jährlich tagt. Soweit es sich um einen politisch brisanten Vorgang handelt, müssen insbesondere die Abgeordneten der Regierungsfraktion ihr Votum im Petitionsausschuss mit ihrer Fraktion sowie mit „ihrer“ Regierung abstimmen, denn schließlich wollen sie keine „politischen Eigentore“ schießen, sondern dem Regierungslager als „Liberos“ zur Seite stehen.

Der erste Thüringer Bürgerbeauftragte Karsten Wilsdorf zog zu derartigen Petitionen das sarkastische Resümee: „Dann haben wir schon längst Frühling, der Schnee ist geschmolzen und die Petition hat sich von selbst erledigt." Er hätte das Problem hingegen schon in einem Telefongespräch mit dem Bürgermeister der Gemeinde oder durch die kurzfristige Verabredung eines „runden Tisches“ mit allen Betroffenen vor Ort sehr schnell lösen können.

18 Anne Debus, a.a.O. (Fn. 13), Einleitung, S. 19, Fn. 5. Eine genaue beispielhafte Auswertung des Jahresberichts 2003 ergab für die Bearbeitungsdauer von 764 Bürgeranliegen folgende, durchaus repräsentative, Bearbeitungszeiten: weniger als 3 Monate (644 Anliegen / 84,3 Prozent), weniger als 6 Monate (85 Anliegen / 11,1 Prozent), mehr als 6 Monate (35 Anliegen / 4,6 Prozent). 


\subsection{Erfolgsbilanzen}

Blickt man - trotz aller erwähnten Schwierigkeiten der Messung - auf die Erfolgsbilanzen beider Institutionen, ergibt sich ein ähnliches Bild zu Gunsten des Bürgerbeauftragten. Er konnte von 2001 bis 2006 im Einvernehmen mit dem Petenten zwischen circa 80 und 94 Prozent der Fälle zu dessen Zufriedenheit erledigen.

Die Fälle, in denen Petenten mit den Entscheidungen des Bürgerbeauftragten nicht einverstanden waren und die deswegen gemäß $\$ 5$ Abs. 1 Satz 3 BüG 2000 an den Petitionsausschuss als quasi zweite Instanz weitergeleitet wurden, lagen zwischen 2001 und 2006 bei einer Gesamtzahl von 4.440 Bürgeranliegen einschließlich Petitionen - nur zwischen zehn und maximal 33.

Ein beispielhafter, aber aus Erfahrung durchaus als repräsentativ anzusehender Vergleich der Erfolgsbilanzen des Bürgerbeauftragten im Vergleich zum Petitionsausschuss aus dem Jahr 2003 ergibt das folgende Bild in Tabelle 1.

\begin{tabular}{|l|c|c|}
\hline Tabelle 1: Erfolgsbilanzen des Bürgerbeauftragten im Vergleich zum Petitionsausschuss 2003 \\
\hline & Bürgerbeauftragter & Petitionsausschuss \\
\hline abgeschlossene Eingaben & 770 & 884 \\
tatsächlich abgeholfen & $104(13,5 \%)$ & $80(9,0 \%)$ \\
durch Auskünfte erledigt & $510(66,2 \%)$ & $438(49,6 \%)$ \\
in sonstiger Weise erledigt & $84(10,9 \%)$ & $125(14,1 \%)$ \\
nicht abgeholfen & $33(4,3 \%)$ & $202(22,9 \%)$ \\
von Prüfung abgesehen & $39(5,1 \%)$ & $39(4,4 \%)$ \\
\hline Quelle: Eigene Zusammenstellung.
\end{tabular}

Somit lag im Jahr 2003 die Erfolgsquote des Bürgerbeauftragen bezogen auf Eingaben, bei denen dem Petitum tatsächlich oder durch Auskünfte (beziehungsweise sonstige Hilfen) entsprochen wurde, bei 79,7 Prozent und beim Petitionsausschuss bei 58,5 Prozent. Die Misserfolgsquoten betrugen dementsprechend beim Bürgerbeauftragten nur 4,3, und beim Petitionsausschuss 22,9 Prozent.

Diese Ergebnisse werden durch einen Vergleich der Erfolgsquoten des rheinland-pfälzischen Bürgerbeauftragten mit jenen des Petitionsausschusses des Deutschen Bundestages tendenziell gestützt. So heißt es in der Begründung des oben genannten Gesetzentwurfs der Bundestagsfraktion von Bündnis 90/Die Grünen unter anderem: „In der Praxis konnte der Bürgerbeauftragte Rheinland-Pfalz durch diesen Erledigungsmodus eine deutlich höhere Erfolgsquote erzielen als der Petitionsausschuss des Deutschen Bundestages. Während im Petitionsausschuss regelmäßig circa sieben Prozent der Eingaben (ohne Auskunftserteilung) voll- beziehungsweise teilweise im Sinne des Petenten geregelt wurden, ist die Quote in Rheinland-Pfalz mit circa 25 Prozent erstaunlich hoch. "19

Die Gründe für die höhere Erfolgsquote des Bürgerbeauftragten im Vergleich zum Petitionsausschuss sind ebenfalls sehr einsichtig: Petitionsausschüsse bearbeiten Petitionen weitgehend in einem schriftlichen, anonymen, bürokratischen Verfahren am "grünen Tisch“. Ortstermine sind die absolute Ausnahme. Zwar spricht der Arbeitsbericht des Thüringer

19 BT-Drs. 13/3578, S. 9. 
Petitionsausschusses zum Beispiel für das Jahr 2010 davon, dass sich der Ausschuss „nicht selten ... zunächst auch selbst vor Ort ein Bild zu dem vorgetragenen Sachverhalt" macht (S. 7), verschweigt aber schamhaft die genaue Zahl von Ortsterminen. Tatsächlich nahm der Petitionsausschuss als Ausschuss im Jahr 2010 keinen einzigen und stellvertretend durch einzelne Abgeordnete lediglich vier Ortstermine wahr; der Bürgerbeauftragte absolvierte hingegen zur Zeit der Rechtslage vor der Reform im Jahr 2007 zum Beispiel im Jahr 2003 allein 44 Termine.

Wer je einen schriftlichen Bescheid einer Behörde erhalten hat und sich im Kontakt mit der Behörde um eine selbst noch so geringfügige Änderung des Bescheids bemüht hat, weiß wie schwer es einer Behörde fällt, eine einmal gefasste Meinung zu revidieren, zumal, wenn sie schon schriftlich vorliegt. Das Beharrungsvermögen von Behörden ist besonders groß, wenn ihre Position zuvor intern mit mehreren Referaten oder Abteilungen abgestimmt und sogar von der nächst höheren Stelle bestätigt worden ist. Verwaltungen weichen von in schriftlichen, formalisierten Verfahren festgezurrten Ergebnissen kaum mehr ab. Daher enthalten die Stellungnahmen der Exekutive selbst in Zweifelsfällen zumeist nur Rechtfertigungen des eigenen Tuns, ohne offene, kreative Flexibilität an den Tag zu legen. Das gilt leider auch für Verwaltungsentscheidungen, die zwar rechtmäßig sind, bei denen es jedoch einen eventuell sogar großen Ermessensspielraum gibt. Regelmäßig fehlt es an der nötigen Offenheit und dem Bemühen, diesen Spielraum gegebenenfalls im Interesse der Petenten einfach nur nochmals auszuloten. Dabei besteht gerade im Bereich des Ermessens über das Petitionsrecht die große Chance, bei aller Wahrung der Rechtsstaatlichkeit den Interessen der Petenten ein Stück entgegen zu kommen.

Die übliche Verfahrenspraxis von Bürgerbeauftragten bietet bei der Bearbeitung von Petitionen demgegenüber erhebliche Vorteile: Ihre Entscheidungen fallen nicht aufgrund schriftlicher Stellungnahmen fern der Praxis; sie haben vielmehr in weit größerem Umfang als das bei den Mitgliedern von Petitionsausschüssen der Fall sein kann, die Möglichkeit, vor Ort zu gehen und alle Betroffenen „Auge in Auge“ an einem „runden Tisch“ zu versammeln und um einvernehmliche Lösungen zu ringen. Von derartigen Ortsterminen wird vom Bürgerbeauftragten in der Praxis auch rege Gebrauch gemacht. Der Bürgerbeauftragte fungiert dabei weitgehend als eine Art „Mediator“. Mediatoren werden bei der Konfliktlösung in Rechts- oder Wirtschaftsangelegenheiten zunehmend erfolgreich hinzugezogen, weil sie eine Atmosphäre schaffen, die die Bereitschaft zum gegenseitigen Zuhören und Verstehen und letztlich auch die Kompromissbereitschaft günstig beeinflusst. Von diesen positiven Erfahrungen kann das Petitionswesen nur bedingt profitieren, denn sie lassen sich realistischerweise nur von Bürgerbeauftragten, nicht jedoch von Ausschüssen nutzen.

Kennzeichnend für das „gesichtslose“ anonyme Verfahren im Petitionsausschuss ist auch die Praxis in Thüringen, dass alle Schreiben an den Petenten, einschließlich des abschließenden Petitionsbescheids, die Unterschrift von Beamten der Parlamentsverwaltung tragen und nicht die des Vorsitzenden des Petitionsausschusses oder die des jeweils für die Petition federführenden Abgeordneten. Demgegenüber steht der Bürgerbeauftragte für seine Entscheidungen mit seinem Namen ein und zeichnet Bescheide folglich auch persönlich.

Ein weiteres Hindernis für eine offene, kreative und flexible Erledigung von Petitionen durch Petitionsausschüsse besteht - wie bereits hervorgehoben - darin, dass auch für das Petitionsverfahren die üblichen politischen Regeln der parlamentarischen Kontrolle gelten. Sie greifen insbesondere dann, wenn politisch relevante Entscheidungen der Exekutive Ge- 
genstand der Petition sind und womöglich noch ein Amtsträger aus dem politischen Lager der Mehrheitsfraktion hinter der vom Petenten angegriffenen Entscheidung steht. Derartige typische, einer effektiven parlamentarischen Kontrolle im Wege stehende Konstellationen lassen sich - wie in anderen parlamentarischen Kontrollverfahren - nur über die Öffentlichkeit aufbrechen. Aber auch daran fehlt es im Petitionsverfahren; die Petitionsausschüsse tagen nicht öffentlich ( $\$ 15$ Abs. 1 PetG, 2007), und die verfahrensmäßig vorgesehenen Möglichkeiten, mit dem Petitionsvorgang den Landtag in öffentlicher Sitzung zu befassen $(\$ 100 \mathrm{GO}$ ThürLT), werden so gut wie nie genutzt. Die Abgeordneten des Regierungslagers blockieren aus den oben genannten Gründen, und der Opposition fehlt es oft an der nötigen Motivation, weil sie meint, damit parlamentarisch kaum punkten zu können. Der Bürgerbeauftragte ist derartigen parlamentarischen Regeln und Usancen nicht unterworfen.

\subsection{Vertrauen der Bürger in den Bürgerbeauftragten}

In unserer hochkomplexen, differenzierten, arbeitsteiligen Gesellschaft bedarf es des Vertrauens der Bürger in ihre demokratisch legitimierten Repräsentanten, damit sie deren Entscheidungen akzeptieren können. ${ }^{20}$

Dieses Vertrauen ist jedoch stark im Schwinden begriffen, gerade auch gegenüber Politikern und Abgeordneten ${ }^{21}$, zumal sie dem Bürger im Petitionsverfahren, wie bereits dargestellt, als Repräsentanten eines anonymen Staatsapparats begegnen. Demgegenüber besitzt ein Bürgerbeauftragter den Vorteil, dass er als „Moderator, Dolmetscher und Lotse an der Schnittstelle zwischen Bürger und Staat " 22 erscheint und somit dem Bürger als individuell personalisierte, kommunizierende Institution mit menschlichem Antlitz entgegentritt, was die Distanz zwischen Staat und Bürger verringert. Der Bürgerbeauftragte vermag die Funktion eines „Mediators“ zu übernehmen, der nicht etwa nur die Interessen der Bürger einseitig im Blick hat, sondern auch um Verständnis und Akzeptanz für staatliche Entscheidungen werben kann. ${ }^{23}$

Der wichtigste immer wieder zu hörende Einwand gegen die Einrichtung eines Bürgerbeauftragten, dass die Bundesrepublik Deutschland „über ein ausgebautes und lückenloses System der Verwaltungsgerichtsbarkeit mit Generalklausel verfügt “24, überzeugt nicht, weil das Hauptbetätigungsfeld im Petitionswesen gerade im Bereich staatlicher Ermessensausübung liegt. Daher vermag ein Bürgerbeauftragter im Hinblick auf die in einigen Ländern - so auch in Thüringen - vollzogene beziehungsweise geplante Abschaffung von Widerspruchsverfahren insoweit eine wichtige Ersatzfunktion wahrzunehmen.

20 Dazu näher Joachim Linck, Zurück zum ehrenamtlichen Landesparlamentarier?, in: Hans Herbert von Arnim (Hrsg.), Defizite in Staat und Verwaltung, Berlin 2010, S. 91 - 98.

21 Vgl. ebenda, S. 93.

22 So der Titel des Aufsatzes von Anne Debus, a.a.O. (Fn. 4).

23 Vgl. ebenda, Einleitung, S. 19 ff.

24 So die Enquetekommission „Verfassungsreform“ des Bundestags in ihrem Schlussbericht, a.a.O. (Fn. 5), S. 64. 


\section{3. „Entmachtung“ des Thüringer Bürgerbeauftragten wegen zu großen Erfolgs?}

Diese im Vergleich zum Petitionsausschuss erheblich erfolgreichere Arbeit des Bürgerbeauftragten war dem Thüringer Landtag, insbesondere seinen Mitgliedern im Petitionsausschuss, ein Dorn im Auge. Doch warum nur? Zur Beantwortung dieser Frage bedarf es eines kritischen Blicks auf den heutigen Status und die sowohl wirtschaftliche als auch finanzielle Situation von Landtagsabgeordneten in allen Flächenländern und somit auch in Thüringen. ${ }^{25}$

Die Landtagsabgeordneten sind staatlich besoldete Berufsabgeordnete, die ganz überwiegend „für und von der Politik leben“ (Max Weber). Ihnen Aufgaben zu entziehen und auf andere Institutionen zu verlagern, hieße, ihre berufliche und damit zugleich wirtschaftliche Existenz zu gefährden. In dieser Situation befanden (und befinden) sich insbesondere die Abgeordneten in den Petitionsausschüssen. Entgegen einer frühen, allerdings nur sehr kurzen Blüte des Petitionswesens in den neuen Bundesländern, als das politische Führungspersonal eingedenk der Erfahrungen mit dem teilweise relativ wirksamen Eingabewesen der DDR in die Petitionsausschüsse drängte ${ }^{26}$, ist inzwischen auch in Thüringen insoweit der westdeutsche Alltag eingezogen: Die Petitionsausschüsse werden vornehmlich mit Hinterbänklern und Nachrückern besetzt. ${ }^{27}$

Die parlamentarische Führungsriege präferiert inzwischen längst andere Ausschüsse, denn mit der Arbeit in Petitionsausschüssen ist, vielleicht abgesehen von derjenigen des Vorsitzenden, kein „politischer Blumentopf“ zu gewinnen. Die Abgeordneten arbeiten dort im Stillen in nicht öffentlichen Sitzungen, und es kostet sogar jedes Mal erhebliche Mühe, ihre jährlichen Berichte in den öffentlichen Plenarsitzungen so zu platzieren, dass sie wenigstens einmal im Jahr von der breiten Öffentlichkeit wahrgenommen werden können.

Diesen politisch nur wenig renommierten Abgeordneten fällt es daher besonders schwer, sich politisch zu profilieren, wodurch ihre Chancen, auf Landeslisten gut abgesichert zu werden, nicht gerade groß sind. Sie gehören - wenigsten in den großen Fraktionen - zum „politischen Präkariat"; umso mehr ist es aus ihrer Sicht nachvollziehbar, dass sie um ihre parlamentarischen Arbeitsplätze kämpfen.

Folglich wurde auf der Grundlage von drei Gesetzentwürfen der damals im Thüringer Landtag vertretenen Fraktionen ${ }^{28}$ das „Thüringer Gesetz über das Petitionswesen“ vom 15. Mai 2007 (PetG 2007) und das „Thüringer Gesetz über den Bürgerbeauftragten“ vom gleichen Tage (BüG 2007) verabschiedet ${ }^{29}$, mit dem der Bürgerbeauftragte in einschneidender Weise entmachtet wurde; gleichzeitig wurden die Kompetenzen des Petitionsausschusses maßgeblich gestärkt. Dieses parlamentarische „rollback“ bestand in erster Linie darin, dem Bürgerbeauftragten die Bearbeitung und Erledigung von Petitionen im Sinne

25 Vgl. dazu eingehend Joachim Linck, a.a.O. (Fn. 20), S. 91 ff.; ders., in: NJW 2008, S. 24; Hans Herbert von Arnim, in: Rudolf Dolzer / Karin Graßhof / Wolfgang Kahl / Christian Waldhoff (Hrsg.), Bonner Kommentar zum Grundgesetz, Art. 48, Rn. 162 ff.

26 Vgl. dazu näher: Joachim Linck, Wie ein Landtag laufen lernte: Erinnerungen eines westdeutschen Aufbauhelfers in Thüringen, Köln / Weimar 2010, S. 157 ff.

27 Vgl. ebenda, S. 157; vgl. auch BT-Drs. 13/3578, S. 7; Thomas Würtenberger, in: Rudolf Dolzer I Karin Graßhof / Wolfgang Kahl / Christian Waldhoff(Hrsg.), Bonner Kommentar zum Grundgesetz, Art. 45c, Rn. 38.

28 Vgl. Drs. 4/2701 (SPD); Drs. 4/2728 (CDU); Drs. 4/2735 (Linkspartei.PDS).

29 Siehe GVBl. 2007, S. 57; GVBl. 2007, S. 54. 
von Artikel 14 ThürLV ausnahmslos zu entziehen. Der Bürgerbeauftragte hat seit dieser Zeit die ihm zugeleiteten Petitionen ,an die zuständige Stelle oder den Landtag weiterzuleiten" ( $\$ 1$ Abs. 3 BüG 2007); das heißt in aller Regel, dass er sie an den Petitionsausschuss übergibt, der sie - nunmehr in ausschließlicher Zuständigkeit - entscheidet ( $\$ 8$ PetG 2007). In die Zuständigkeit des Bürgerbeauftragten fallen seitdem nur noch „Bürgeranliegen“, die keine Petitionen im Sinne des $\$ 1$ Thüringer Petitionsgesetz sind ( $\$ 1$ Abs. 1, Satz 2 i.V.m. Abs. 2 Nr. 1 BüG 2007).

\section{Grundsätzliche rechtspolitische Forderung nach Einführung starker Bürgerbeauftragter}

Die geltende Rechtslage bedarf aus rechtspolitischen Gründen, insbesondere aus der Sicht der Bürger, einer grundlegenden Reform. Ziel muss es sein, Bürgerbeauftragte einzuführen, deren Kompetenzen erheblich zu stärken sind.$^{30}$ Die rechtspolitische Forderung stützt sich zwar nur auf die dargelegten Erfahrungen in Thüringen, wo es zeitweise zwischen Petitionsausschuss und Bürgerbeauftragtem eine echte Konkurrenz gegeben hat; aber der Effizienzvergleich fällt so eindeutig und nachvollziehbar zu Gunsten des Bürgerbeauftragten aus, dass es gerechtfertigt ist, diese Erfahrungen für den Bund und die anderen Länder nutzbar zu machen.

Bürgerbeauftragte sollten daher im Bund und in allen Ländern für die Bearbeitung und Entscheidung aller individuellen Petitionen zuständig sein. Dafür müssen ihnen auch die dazu erforderlichen Befugnisse zustehen, um die Petitionen zeitnah und erfolgreich bearbeiten zu können. Die Begründung dafür ist - wie bereits oben im Einzelnen dargelegt - so einfach wie zwingend:

Erstens ist die Bearbeitungszeit für Petitionen durch Bürgerbeauftragte ganz erheblich kürzer als bei Petitionsausschüssen; zweitens ist auch die Erfolgsbilanz von Bürgerbeauftragten weitaus besser als die von Petitionsausschüssen. Orientiert man sich bei einer Reform des Petitionswesens an den Interessen der Bürger, kann es überhaupt keine Zweifel geben: Die primäre Zuständigkeit für die Bearbeitung und Entscheidung über individuelle Petitionen muss bei Bürgerbeauftragten liegen. Sollte die Einführung von starken Bürgerbeauftragten dennoch unter dem maßgeblichen Einfluss von Mitgliedern der Petitionsausschüsse blockiert werden, würde sich bei derartigen Entscheidungen in eigener Sache die Frage stellen, ob die Bürger selbst im Wege von Volksbegehren und Volksentscheiden die Einführung von Bürgerbeauftragten erzwingen sollten.

\section{Zur Ausgestaltung des Status und der Bestellung von Bürgerbeauftragten}

Bürgerbeauftragte können die ihnen nach den bisherigen Ausführungen zugedachte Funktion nur erfolgreich erfüllen, wenn sie den verfassungsrechtlich gesicherten Status von un-

30 Meine skeptischen Bemerkungen gegenüber der Einführung eines Bürgerbeauftragen (vgl. dazu Joachim Linck, in: Joachim Linck / Siegfried Jutzi / Jörg Hopfe, Die Verfassung des Freistaats Thüringen, Stuttgart u.a. 1994, Art. 65, Rn. 8) halte ich aufgrund neuer zwischenzeitlich gewonnener Erfahrungen mit dem Thüringer Bürgerbeauftragten in der Zeit zwischen 2001 und 2006 nicht mehr aufrecht. 
abhängigen Amtsträgern erhalten, durch die Art ihrer Bestellung das nötige Vertrauen der Bürger genießen und die erforderlichen eigenständigen Befugnisse besitzen, um ihre Aufgaben effizient erfüllen zu können.

\subsection{Unabhängiger Status}

Die einem Richter vergleichbare Unabhängigkeit von Bürgerbeauftragten sowohl gegenüber der Landesregierung als auch dem Landtagspräsidenten sollte in der Landesverfassung garantiert werden, wie dies zum Beispiel in Art. 36 Abs. 2 Satz 1 der Verfassung von Mecklenburg-Vorpommern geschehen ist.

\subsection{Bestellung von Bürgerbeauftragten}

Die Bestellung von Bürgerbeauftragten sollte in einer Weise erfolgen, dass sie das erforderliche Vertrauen der Bürger in möglichst hohem Maße genießen; das Amt darf keinesfalls als Abschiebebahnhof beziehungsweise Versorgungsposten für ansonsten unerwünschte Amtsträger missbraucht werden. Damit verbietet sich von vornherein jegliche Beteiligung der Landesregierungen, da sie als verantwortliche Spitze der Exekutive in erheblichem Umfang Objekt der Kontrolle durch das Petitionsrecht sind.

Erwägenswert wäre auch eine Wahl von Bürgerbeauftragten durch die Parlamente. Dafür sollte dann allerdings zumindest eine Zweidrittelmehrheit vorgesehen werden, da andernfalls das politische Lager aus Regierung und Mehrheitsfraktion(en) einen ihnen gegenüber geneigten, eventuell sogar willfährigen Kontrolleur bestimmen könnte. Eine derartige Lösung würde aber dennoch - wie bei allen parlamentarischen Wahlen mit einer vorgeschriebenen Zweidrittelmehrheit - problematisch sein, weil im Vorfeld derartiger Wahlen üblicherweise sachfremde Kompensationsgeschäfte zwischen den Fraktionen zur Absicherung der Zweidrittelmehrheit abgesprochen werden.

Eindeutig vorzuziehen wäre daher eine Direktwahl von Bürgerbeauftragten durch das Volk, weil damit deren Unabhängigkeit sowie das Vertrauen der Bürger in „ihren“ Bürgerbeauftragten erheblich gestärkt würden.

Die erforderliche Unabhängigkeit von Bürgerbeauftragten sollte - ähnlich wie bei den Bundesverfassungsrichtern - zusätzlich dadurch gestärkt werden, dass ihre Wahlperioden - ohne eine Wiederwahlmöglichkeit - zum Beispiel zehn Jahre betragen; allerdings müsste dabei auch folgerichtig mit den nötigen Quoren eine Abwahlmöglichkeit vorgesehen werden.

\subsection{Befugnisse von Bürgerbeauftragten}

Bürgerbeauftragten sollten unmittelbar, also nicht etwa abgeleitet vom Petitionsausschuss, die Befugnisse zur Aufklärung von Petitionsvorgängen zustehen, die Petitionsausschüsse nach geltendem Recht besitzen (vgl. zum Beispiel Art. 65 Abs. 2 ThürLV).

Da Bürgerbeauftragte einerseits aufgrund des Gewaltenteilungsprinzips nicht selbst unmittelbar in verpflichtender Weise in die Exekutive eingreifen und schon gar nicht anstelle 
der Exekutive Verwaltungsentscheidungen zugunsten von Petenten treffen dürfen ${ }^{31}$, aber andererseits auch nicht nur auf schlichte Bitten beziehungsweise Ersuchen an die Exekutive angewiesen sein sollten (so aber $\$ 4$ BüG 2007), wäre ihnen ein gewisses Druckpotential zu eröffnen, um ihren argumentativen Einfluss auf die Exekutive zu stärken. Das effektivste Mittel zur Stärkung einer Kontrolltätigkeit ist auch hier - wie auch sonst bei der parlamentarischen Kontrolle - die Öffentlichkeit. ${ }^{32}$ Den Bürgerbeauftragten sollte daher ein Rederecht im Parlament zugestanden werden, um notfalls in öffentlicher Sitzung Druck auf die Regierung für die Verwirklichung der von ihnen empfohlenen Erledigungen von Petitionen ausüben zu können. Da aufgrund dieser Möglichkeit zumindest der Rechtfertigungsdruck erhöht würde, ginge allein schon von der Befugnis, im Parlament reden zu können, eine nicht unerhebliche Wirkung auf die Regierung aus („fleet in being“), bereits im Vorfeld zu einvernehmlichen Regelungen zu gelangen.

\section{Verbleibende Aufgaben für Petitionsausschüsse}

Petitionsausschüsse würden mit der oben vorgeschlagenen Konzeption zugunsten von Bürgerbeauftragten nicht ihre Existenzberechtigung und Bedeutung verlieren. Vielmehr sollten ihnen weiterhin alle so genannten Legislativpetitionen im weiteren Sinne zur ausschließlichen Bearbeitung und Entscheidung überlassen werden. ${ }^{33} \mathrm{Zu}$ dieser Art von Petitionen sollten nicht nur nach $\$ 1$ Abs. 2 Satz 2 Petitionsgesetz 2007 „Vorschläge zur Gesetzgebung" gehören, sondern auch Vorschläge zur Setzung anderer Rechtsnormen wie zum Beispiel Verordnungen oder Satzungen; auch darauf können Parlamente durch schlichte Parlamentsbeschlüsse politisch-empfehlend Einfluss nehmen.

Die Rechtsetzung ist eine genuine Aufgabe eines Parlaments. Hier werden die pluralistischen Interessen des Volkes diskutiert, gebündelt und integriert. Folglich müssen sich Petenten mit Anregungen zur Reform von Rechtsvorschriften unmittelbar und ausschließlich an den Petitionsausschuss wenden können. In diesen Fällen stellt sich rein strukturell eine völlig andere Situation als bei der Bearbeitung von Individualbeschwerden. Sollte allerdings Bürgerbeauftragten bei der Bearbeitung von Petitionen die Notwendigkeit zur Änderung bestimmter Rechtsvorschriften auffallen, sollte es auch ihre Aufgabe sein, das Parlament auf den Reformbedarf hinzuweisen und ihm gegebenenfalls auch Reformvorschläge zu unterbreiten.

Weiterhin gibt es gute Gründe, auch Massen- und Sammelpetitionen (vgl. für den Bund Nr. 2.2 und 8.4 der Grundsätze des Petitionsausschusses sowie $\$ 14$ Thür PetG) in die ausschließliche Kompetenz des Petitionsausschusses zu geben, weil es sich dabei in aller Regel nicht um individuelle Anliegen von Bürgern, sondern um allgemeine politische Angelegenheiten handelt, die eine politische Reaktion erfordern.

Fraglich ist, ob Petitionsausschüsse darüber hinaus auf einen entsprechenden Antrag eines mit der Entscheidung eines Bürgerbeauftragten unzufriedenen Petenten quasi als eine zweite (Beschwerde)-Instanz Petitionsentscheidungen des Bürgerbeauftragten aufheben

31 So die einhellige Meinung, vgl. ebenda, Art. 65, Rn. $14 \mathrm{f}$.

32 Vgl. Joachim Linck, Die Parlamentsöffentlichkeit, in: ZParl, 23. Jg. (1992), H. 4, S. 673 - 708, S. 674 m.w.N.

33 In diesem Sinne $\$ 102$ Abs. 2 GOLT Rheinland-Pfalz. 
oder ändern und letztinstanzlich entscheiden sollten. Dagegen spricht das bereits oben erörterte Argument, dass auf diese Weise in die Petitionsentscheidungen durch die Dominanz der Regierungslager parteipolitische Erwägungen einfließen könnten, was im Sinne des Gemeinwohls im Petitionsverfahren schädlich wäre. Die sachorientierte, neutrale Arbeit von Bürgerbeauftragten liefe damit Gefahr, konterkariert zu werden; außerdem könnten dadurch Ansehen und Autorität von unmittelbar demokratisch legitimierten Bürgerbeauftragten beschädigt werden.

\section{Ein positiver Nebeneffekt: Einsparung von Kosten}

Eine parallele Bearbeitung von Petitionen durch Petitionsausschüsse und Bürgerbeauftragte mit Hilfe der ihnen jeweils angegliederten Verwaltungsapparate verursacht unnötige Kosten. Nach der hier vorgeschlagenen Konzeption würden Petitionsausschüsse erheblich weniger Personal benötigen. Für die ihnen verbleibenden Legislativpetitionen sowie die Massen- und Sammelpetitionen wäre eine Betreuung durch die sonstigen Ausschussassistenten der Parlamentsverwaltungen ausreichend. So könnte zum Beispiel der derzeitige Geschäftsbereich des Petitionsausschusses in Thüringen mit insgesamt zehn Stellen fast vollständig abgebaut und die Verwaltung des Bürgerbeauftragten um einige Stellen aufgestockt werden. Die Einsparung von Personalkosten sollte in einer Zeit, in der der Abbau von Schulden dringlich ist, nicht gering geschätzt werden.

\section{Zusammenfassung der rechtspolitischen Empfehlungen zur Einführung von starken Bürger- beauftragten}

Die Thüringer Erfahrungen sollten zu einer ausgiebigen Diskussion über die Einführung starker Bürgerbeauftragter anregen. Dazu seien die zentralen Empfehlungen noch einmal zusammengefasst:

(1) Bürgerbeauftragte sollten grundsätzlich alle Petitionen bearbeiten und entscheiden, mit Ausnahme der Legislativpetitionen sowie der Massen- und Sammelpetitionen.

(2) Die Legislativpetitionen sowie die Massen- und Sammelpetitionen sollten weiterhin in die - insoweit ausschließliche - Kompetenz der Petitionsausschüsse fallen.

(3) Bürgerbeauftragte sollten einen unabhängigen Status erhalten, der verfassungsrechtlich abzusichern wäre. Weiterhin sollten ihre Befugnisse entsprechend denjenigen von Petitionsausschüssen in den Verfassungen verankert werden.

(4) Bürgerbeauftragte sollten unmittelbar vom Volk gewählt werden. 